\title{
Weak-winner phase synchronization: A curious case of weak interactions
}

\author{
Anshul Choudhary $\odot,{ }^{1,2}$ Arindam Saha, ${ }^{1,3}$ Samuel Krueger, ${ }^{4}$ Christian Finke, ${ }^{1}$ Epaminondas Rosa Jr. $\odot,{ }^{4}$ \\ Jan A. Freund, ${ }^{1}$ and Ulrike Feudel $\odot^{1}$ \\ ${ }^{1}$ Theoretical Physics/Complex Systems, ICBM, Carl von Ossietzky University of Oldenburg, 26129 Oldenburg, Germany \\ ${ }^{2}$ Nonlinear Artificial Intelligence Laboratory, North Carolina State University, Raleigh, North Carolina 27695, USA \\ ${ }^{3}$ Department of Computer Science, University of Sheffield, Sheffield S10 2TN, United Kingdom \\ ${ }^{4}$ Department of Physics and School of Biological Sciences, Illinois State University, Normal, Illinois 61790, USA
}

(Received 11 September 2020; accepted 5 April 2021; published 24 May 2021)

\begin{abstract}
We report the observation of a nontrivial emergent state in a chain of nonidentical, heterogeneously coupled oscillators where a set of weakly coupled oscillators becomes phase synchronized while the strongly coupled ones remain drifting. This intriguing "weak-winner" synchronization phenomenon can be explained by the interplay between nonisochronicity and the natural frequency of the oscillator, as coupling strength is varied. Furthermore, we present sufficient conditions under which the weak-winner phase synchronization can occur for limit cycles as well as chaotic oscillators. Employing a model system from ecology as well as a paradigmatic model from physics, we demonstrate that this phenomenon is a generic feature for a large class of coupled oscillator systems. The realization of this peculiar, yet quite generic weak-winner dynamics can have far-reaching consequences in a wide range of scientific disciplines that deal with the phenomenon of phase synchronization, including synchronization of networks. Our results also highlight the role of nonisochronicity (shear) as a fundamental feature of an oscillator in shaping emergent dynamical patterns in complex networks.
\end{abstract}

DOI: 10.1103/PhysRevResearch.3.023144

\section{INTRODUCTION}

Interactions play a fundamental role in nature since many functions, for instance, sensory or information processing, rely on collective tasks, involving an exchange of matter or energy, rather than on individual entities. One of the oldest examples of such collective behavior has originated from the physics of coupled pendulum clocks, which are able to synchronize their motion in time through a weak mechanical coupling [1]. Since its discovery, synchronization has been observed and studied in many areas of science with problems ranging from collective behavior of a large population of chemical oscillators [2] as well as spiking and bursting of neurons in neural networks $[3,4]$ to coupled superconducting Josephson arrays [5] and information transfer in neural systems [6], among others (see Ref. [7] and references therein). Mutual synchronization implies the emergence of coherence in the system through the adjustment of internal rhythms of individual entities without the presence of any central point of control. Several interesting classifications of this broad phenomenon have emerged through extensive research done in the last few decades, namely, complete synchronization (CS) [8], generalized synchronization (GS) [9], and phase synchronization (PS) [10]. CS implies that the coupled systems

Published by the American Physical Society under the terms of the Creative Commons Attribution 4.0 International license. Further distribution of this work must maintain attribution to the author(s) and the published article's title, journal citation, and DOI. remain in step with each other for all times after transients. However, CS can only occur in a system of coupled identical units. By contrast, GS is a state where the coupled elements maintain a functional relationship with each other for all times after transients. Note that GS can be realized for systems where nonidentical units are coupled. In this paper our focus is on the phenomenon of phase synchronization (PS) in coupled systems, which is characterized by oscillators keeping their phases in step with each other while showing no correlation between their amplitudes [10]. It is one of the most ubiquitous phenomena in coupled oscillator systems, pervading both the natural and technological world (see Ref. [11] and references therein). One of the central problems concerning PS is to explain the mechanism(s) behind its emergence for different dynamical behaviors such as limit cycle oscillations, quasiperiodicity, and chaos and also for different coupling topologies such as ring, star, and small-world networks [12]. The contemporary approach essentially relies on the fact that PS emerges out of the complex interplay between coupling and frequency detuning [13-15]. However, in this paper we present an intriguing type of PS which cannot be explained by the aforementioned approach. This state, which we call "weak winner," is an emergent dynamical pattern in a chain of heterogeneously coupled oscillators where the weakly linked part of the chain exhibits phase synchrony while the strongly coupled part remains incoherent. Furthermore, we suggest a mechanism utilizing the concept of nonisochronicity [16-22] to explain the emergence of this nontrivial state of PS.

Formally, two coupled oscillators can be considered phase synchronized if $\Delta \varphi=\left|\varphi_{1}-\varphi_{2}\right|<$ const for sufficiently long periods of time. Here, $\varphi_{1}$ and $\varphi_{2}$ are the phases of the two 


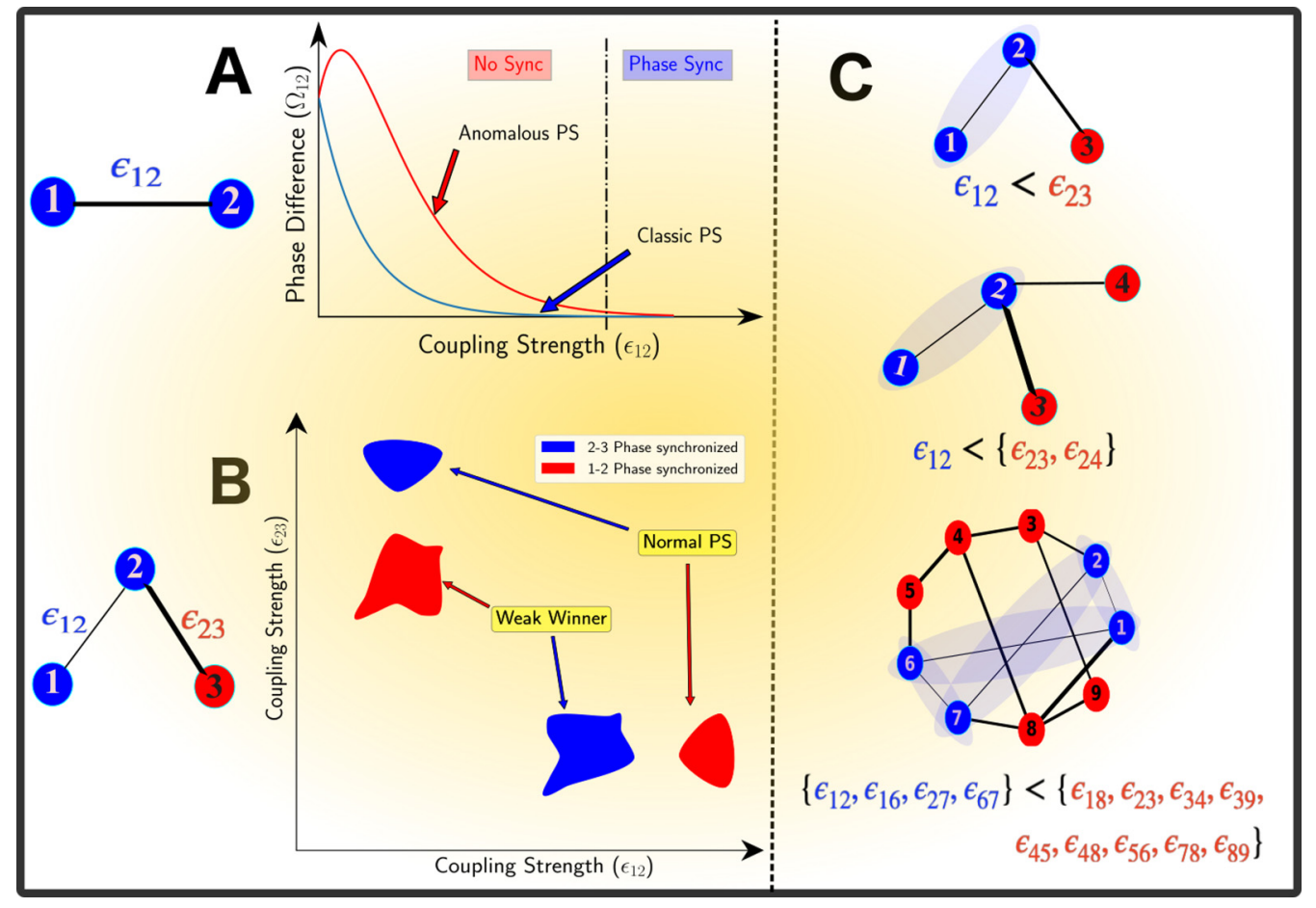

FIG. 1. A brief summary of the essence of the weak-winner phenomenon and how it can manifest in progressively larger networks. (a) depicts the two distinct routes to phase synchronization in a pair of coupled oscillators. One is the classic monotonic decay (blue curve) in phase difference, and the other is anomalous phase synchronization with nonmonotonic decay (red curve) of the phase difference [16-20,36] as coupling is increased. (b) shows how "weak-winner" synchronization emerges in a chain of three heterogeneously coupled oscillators. For three coupled oscillators, there are at least two links, and one of them could be a weaker one; the weakly linked pair of oscillators synchronize their phases, while the other pair with stronger coupling strength remains drifting (details discussed in Sec. III). This phenomenon, where a subset of the network with weakly linked nodes synchronizes their phases while the rest of the network with strongly linked nodes remains drifting, is termed "weak winner." (c) shows some examples of weak-winner phase synchronization as the network size is increased. The coupling strength between oscillators is reflected by the edge thickness. The oscillators colored blue are in phase synchrony. Sync, synchronization.

oscillators, and the constant, for the purpose of our study, is, say, $2 \pi$. Conversely, phase synchrony breaks down whenever one of the oscillators advances its phase at least a full $2 \pi$ cycle ahead of the other [23]. In general, increasing the coupling strength between several oscillators synchronizes their phases. Nonetheless, we show here that surprisingly, the phase synchronization can also appear in the weakly coupled part of the network while the strongly coupled part remains desynchronized (see Fig. 1).

We first demonstrate this using a minimalistic setup with three coupled oscillators. One of the oscillators (say, oscillator 2) is coupled bidirectionally to the two other oscillators (say, oscillators 1 and 3) with coupling parameters $D_{12}$ and $D_{23}$, respectively, and there is no direct coupling between oscillators 1 and 3 (see Fig. 2). This linear chain setup has been studied, for example, in the context of Rössler systems [24] and chaotic lasers [25] forced by two sinusoidal signals, three coupled Rössler systems exhibiting partial phase synchronization [26] and competing synchronization [27], and three coupled semiconductor lasers as well as three neurons displaying relay synchronization [28].

We observe both competing and relay PS [29,30] in our three-oscillator system and, in addition, a counterintuitive type of phase synchronization. The latter happens for certain regions in the $D_{12}-D_{23}$ parameter space, where the two weakly coupled oscillators do stay in phase synchrony with each other while the two strongly coupled oscillators do not. This unexpected behavior, which we call "weak-winner phase synchronization," can be understood as a result of the complex interplay between shear (nonisochronicity) and the natural frequency of individual oscillators as the coupling strength is varied. We also present sufficient conditions under which a coupled oscillator system can exhibit weakwinner PS. Furthermore, we claim that this phenomenon is a generic feature of a large class of coupled nonlinear oscillator systems and provide examples which validate our claim.

\section{MODEL AND FIRST OBSERVATIONS}

To demonstrate the variety of possible applications of weak-winner PS, we first use an example from theoretical ecology to discuss the case of chaotic PS.

Chaotic oscillator model. We consider three coupled chaotic oscillators $(i=1,2,3)$, each of which represents a food chain with three trophic levels at a particular spatial location (patch). This model was originally developed to demonstrate phase synchronization in population dynamics [31]. Each of the three population patches consists of nutrients (vegetation) $x_{i}$, prey (herbivores) $y_{i}$, and predators (carnivores) $z_{i}$ as species. The coupling between the patches accounts for 

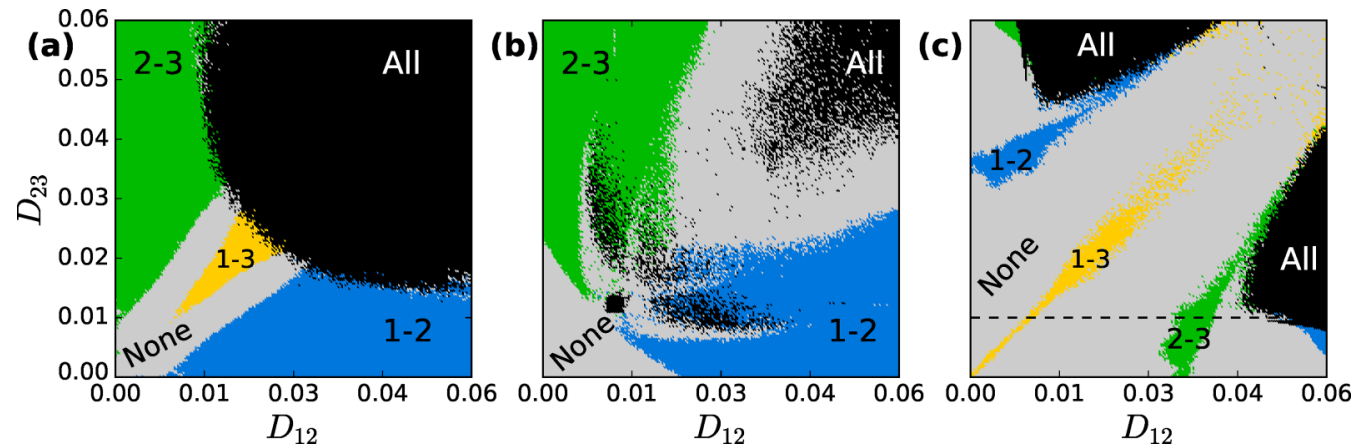

FIG. 2. Parameter space plots showing, as indicated by the labels (and colors), regions of $D_{12}$ and $D_{23}$ values for oscillator pairs in phase synchrony or not, with (a) $b_{2}=1.00$, (b) $b_{2}=1.03$, and (c) $b_{2}=1.1$.

possible migration of herbivores and carnivores. Hence the dynamics of the entire system is given as

$$
\begin{aligned}
& \dot{x}_{i}=a_{i} x_{i}-\frac{\epsilon_{1} x_{i} y_{i}}{\left(1+k_{1} x_{i}\right)}, \\
& \dot{y}_{i}=-b_{i} y_{i}+\frac{\epsilon_{1} x_{i} y_{i}}{\left(1+k_{1} x_{i}\right)}-\epsilon_{2} y_{i} z_{i}+\sum_{j=1}^{3} D_{i j}\left(y_{j}-y_{i}\right), \\
& \dot{z}_{i}=-c_{i}\left(z_{i}-\zeta_{i}\right)+\epsilon_{2} y_{i} z_{i}+\sum_{j=1}^{3} D_{i j}\left(z_{j}-z_{i}\right),
\end{aligned}
$$

where $a_{i}$ represents the vegetation growth rate and $b_{i}$ and $c_{i}$ represent the herbivore and carnivore mortality rates in the absence of interspecies interactions, respectively. The terms $\frac{\epsilon_{1} x_{i} y_{i}}{\left(1+k_{1} x_{i}\right)}$, denoting vegetation-herbivore interaction (prey growth rate), and $\epsilon_{2} y_{i} z_{i}$, describing herbivore-predator interaction, are the standard Holling type II and Lotka-Volterra functions, respectively. The parameter $\zeta_{i}$ accounts for the availability of food for the predator in addition to its preferred prey [32]. Parameters $D_{i j}=D_{j i}$ represent the coupling strength between patches $i$ and $j$ representing the migration of herbivores and carnivores between the patches. For this study, we assume that the three patches are connected in a linear chain, which results in a coupling matrix $\mathbf{D}=$ $\left.\begin{array}{ccc}0 & D_{12} & 0 \\ \left(D_{12}\right. & 0 & D_{23} \\ 0 & D_{23} & 0\end{array}\right)$. We fix the parameters at $a_{1}=a_{2}=a_{3}=$ $1.0, \quad b_{1}=b_{2}=b_{3}=1.0, \quad c_{1}=c_{2}=c_{3}=10.0, \quad \epsilon_{1}=0.25$, $\epsilon_{2}=1.0, k_{1}=0.05$, and $\zeta_{1}=\zeta_{2}=\zeta_{3}=0.006$, unless specified otherwise. For this parameter set, the population densities exhibit chaotic oscillations which resemble those of the Rössler system [33] with phase coherent dynamics.

This means that the trajectory oscillates chaotically around a fixed center of rotation, and on a two-dimensional projection of the attractor, an instantaneous phase can be defined as the increasing angle between an arbitrarily fixed reference axis and the radius of the trajectory [34].

All numerical simulations presented here were performed with the Dormand-Prince (DOPRI5) adaptive step size algorithm [35]. To detect 1:1 phase synchrony between oscillators $i$ and $j$, we compute their unwrapped instantaneous phases $\varphi_{i}(t)$ and $\varphi_{j}(t)$ and check for

$$
\delta \varphi_{i j}=\operatorname{std}\left(\left|\varphi_{i}(t)-\varphi_{j}(t)\right|\right)<2 \pi, \quad \forall t>t_{\text {trans }},
$$

where $\operatorname{std}(\cdot)$ is the standard deviation and the transient time $t_{\text {trans }}$ is taken to be $10^{6}$ arbitrary time units. The choice of using standard deviation as opposed to phase locking value $\left(\mid \varphi_{i}(t)-\right.$ $\left.\varphi_{j}(t) \mid\right)$ for measuring phase synchrony is purely arbitrary, and we have also validated all our results with fixed $(<2 \pi)$ phase locking value.

To study how the coupling strengths (migration rates) affect the phase dynamics among the three oscillators, we generate plots in coupling parameter space indicating different synchronous behaviors (Fig. 2). The values of $D_{12}$ and $D_{23}$ vary in the range between 0.00 and 0.06 . We keep $b_{1}=$ $b_{3}=1.00$ in all three plots and use $b_{2}=1.00, b_{2}=1.03$, and $b_{2}=1.1$ in Figs. 2(a), 2(b), and 2(c), respectively, indicating that environmental conditions for the herbivores are identical in the outer two patches but differ in the central one. In fact, a small increase in the prey mortality parameter $b_{i}$ causes a slight increase in the intrinsic frequency of the $i$ th oscillator. Figure 2(a), representing the case of three coupled identical oscillators, conspicuously displays five different parameter regions characterized by different states of phase synchrony among oscillators: (i) synchronous behavior between oscillators 1 and 2 only, labeled "1-2" (shown in blue), (ii) synchronous behavior between 2 and 3 only, labeled " $2-3$ " (shown in green), (iii) no synchronization between any pair of oscillators, labeled "None" (shown in gray), (iv) relay synchronization between the two outer oscillators 1 and 3, labeled "1-3" (shown in yellow), and (v) phase synchronization of all three oscillators, labeled "All" (shown in black).

The size and location of the synchronization regions change when we increase the $b_{2}$ value to 1.03 [Fig. 2(b)]. We now see that the None synchronized region is enlarged at the expense of complete synchronization, while the 1-2 and 2-3 synchronized regions are not significantly affected.

The original phase structure [Fig. 2(a)] gets some distortion while still maintaining its symmetry. Note that relay synchronization disappears completely in this case. As we advance the $b_{2}$ value further to 1.1 [Fig. 2(c)], all five different parameter regions found in Fig. 2(a) are also present, with two regions of particular interest. Notice in the upper left quadrant the 1-2 synchronized (blue) region for weak $D_{12}$ coupling and strong $D_{23}$ coupling. Due to the stronger $D_{23}$ coupling, this parameter region would be expected to generate 2-3 phase synchronization, not 1-2 as it does. Analogously, due to the symmetry in our setup we find a 2-3 phase synchronization region with strong $D_{12}$ and weak $D_{23}$ 

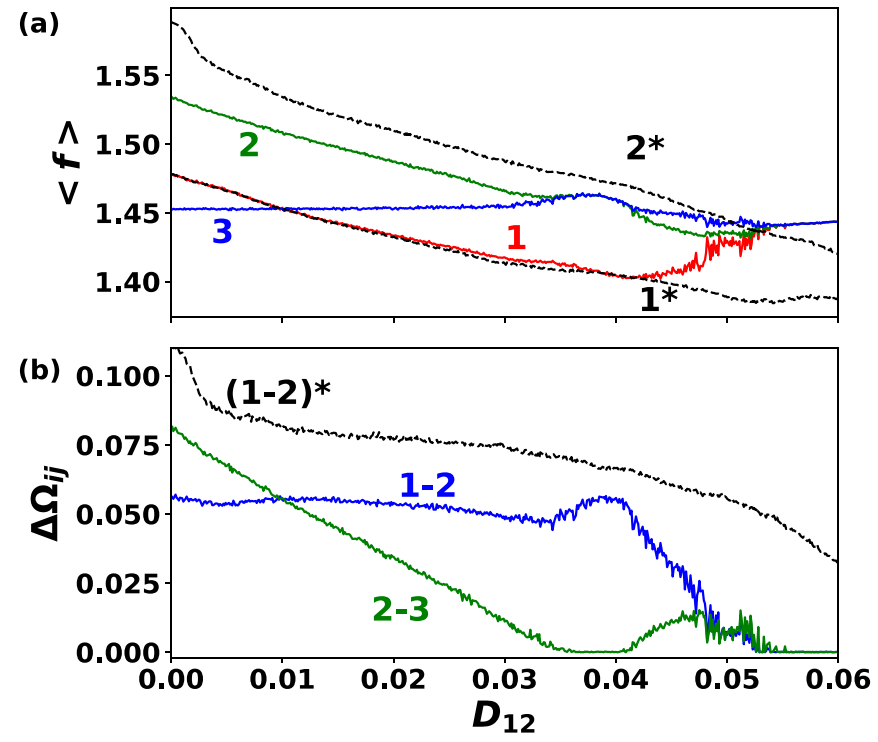

FIG. 3. (a) Variation of mean frequency with coupling strength $D_{12}$ for $b_{2}=1.1$. Corresponding to (a), (b) represents the variation of mean relative frequency for oscillator pairs 1-2 and 2-3, as labeled, with $D_{12}$ for a fixed value of $D_{23}=0.01$ [along the horizontal dashed line in Fig. 2(c)]. For comparison, curves $1 *$ and $2 *$ correspond to the case when $D_{23}=0$.

coupling. We call this phenomenon a weak-winner phase synchronization.

This seemingly counterintuitive PS, where the weak coupling wins over the strong coupling for synchrony, can be corroborated by observing how the mean frequencies of the oscillators $\left\langle f_{1}\right\rangle,\left\langle f_{2}\right\rangle$, and $\left\langle f_{3}\right\rangle$ and their mutual differences, $\Delta \Omega_{i j}=\left|\left\langle f_{i}\right\rangle-\left\langle f_{j}\right\rangle\right|$, vary with changes in the coupling strength. To be specific, we fix $D_{23}=0.01$ and vary $D_{12}$ in the interval [0, 0.06], as indicated by the horizontal dashed line in Fig. 2(c). The mean frequencies are depicted in Fig. 3(a) by the curves labeled 1, 2, and 3, respectively, corresponding to the case exhibiting weak-winner PS [Fig. 2(c)]. Initially separated and distinct, the curves evolve for increasing values of $D_{12}$, showing a tendency for $\left\langle f_{1}\right\rangle$ and $\left\langle f_{2}\right\rangle$ to decrease and for $\left\langle f_{3}\right\rangle$ to remain about constant, corresponding to the case of weak and constant $D_{23}=0.01$ with growing $D_{12}$. When $\left\langle f_{2}\right\rangle$ and $\left\langle f_{3}\right\rangle$ become equal, weak-winner phase synchrony appears. Interestingly, systems 1 and 2 synchronize more easily when system 3 is coupled to system 2, as opposed to the case when $D_{23}=0$ denoted by curves $1 *$ and $2 *$. In fact, system 3 works as a catalyst, causing systems 1 and 2 to synchronize earlier, i.e., for smaller $D_{12}$ values compared with the case when system 3 is not part of the process.

So far, we have observed that due to some interplay between coupling and frequency mismatch, one could get a very unexpected synchronized state - the weak-winner phase synchronization. At first sight, the emergence of weak-winner PS might appear to be the consequence of a phenomenon known as short-wavelength bifurcation (SWB) [36]. However, this is not the case as explained in Appendix D. Instead, we can explain the mechanism of the emergence of such a synchronized state as a result of the existence of anomalous phase synchronization (APS) [16,18-21,37,38]. To demonstrate this in detail, we recall briefly the concept of APS. For a system of two coupled oscillators, APS is a state wherein the frequency difference between the oscillators shows a nonmonotonic behavior with respect to the coupling strength [39-42]. Instead of monotonically decreasing, the frequency difference increases for a certain coupling range before its inevitable decay with increasing coupling strength (see Appendix $\mathrm{C}$ for the intuitive understanding behind APS).

This nonmonotonic relationship between coupling and the spread of frequencies occurs when $\operatorname{Cov}\left(\omega_{i}, q_{i}\right)>0$, where

$$
\operatorname{Cov}\left(\omega_{i}, q_{i}\right)=\left(\omega_{i}-\left\langle\omega_{i}\right\rangle\right)\left(q_{i}-\left\langle q_{i}\right\rangle\right)
$$

and $\omega_{i}$ and $q_{i}$ are the natural frequency and shear (nonisochronicity) of the $i$ th oscillator [16]. Now, for our system [Eqs. (1a)-(1c)], we do see the signatures of APS as shown in Fig. 3(b), where the frequency difference of oscillator pair 1-2 varies nonmonotonically with coupling. However, to fully analyze the system, one must have a clear definition of shear in the system. Generally, both shear $q$ and natural frequency $\omega$ are functions of the system parameters, and in order to check the $\operatorname{Cov}[\omega, q]$, these functions need to be determined. While it is possible to approximate these functions numerically for any nonlinear oscillator, we find it more convincing to study a paradigmatic system which has both shear and natural frequency explicitly present in the governing equations as system parameters.

\section{MECHANISM: A PARADIGMATIC MODEL APPROACH}

To explore the mechanism of the emergence of weakwinner PS, we turn to a simpler model which is known to exhibit APS and which also contains frequency and shear as system parameters.

Limit cycle model. Here, we are going to use the same coupling structure as before but with individual oscillators represented by complex Stuart-Landau equations. The StuartLandau equation represents a generic mathematical equation describing the behavior of any nonlinear oscillator close to the onset of oscillations. Therefore, in this system the oscillators exhibit only limit cycles when uncoupled and no chaotic oscillations. Interestingly, the extension of the Stuart-Landau equation to spatial domains is given by the complex GinzburgLandau equation, which is one of the most widely studied nonlinear equations in the physics community, describing a plethora of phenomena ranging from superconductivity [43] and Bose-Einstein condensation [44] to nonlinear waves and chemical oscillations [45].

The governing dynamics of the Stuart-Landau system is determined by

$$
\dot{z}_{j}=z_{j}\left[1+i\left(\omega_{j}+q_{j}\right)-\left(1+i q_{j}\right)\left|z_{j}\right|^{2}\right]+\sum_{k=1}^{3} D_{j k}\left(z_{k}-z_{j}\right)
$$

where $z_{j}=\rho_{j} e^{i \theta_{j}}$ and $j=1,2,3$. Here, $\omega_{j}$ represents the intrinsic frequency of the oscillator $j$, and $q_{j}$ is the degree of nonisochronicity (or shear), which is basically a measure of the dependence of the frequency on the amplitude of the oscillator. In this model, shear and frequency are system 
parameters. In order to understand the phase dynamics of the system, we reduce Eq. (4) to a pure phase equation that is valid in the weak coupling limit, given by

$$
\dot{\theta}_{j}=\omega_{j}+q_{j} \sum_{k=1}^{3} D_{j k}+\sum_{k=1}^{3} D_{j k}\left[\sin \phi_{j k}-q_{j} \cos \phi_{j k}\right],
$$

where $\phi_{m n}=\theta_{n}-\theta_{m}$ is the relative phase between oscillators $m$ and $n$. Equation (5) can be further represented in terms of the evolution of relative phases as

$$
\begin{aligned}
& \dot{\phi}_{12}=C_{1}-A_{1} \sin \left(\phi_{12}+\alpha\right)-B_{1} \sin \left(\phi_{32}+\beta\right), \\
& \dot{\phi}_{32}=C_{2}-A_{2} \sin \left(\phi_{12}+\beta\right)-B_{2} \sin \left(\phi_{32}+\alpha\right),
\end{aligned}
$$

with the constants $C_{1}=\Delta \omega+D_{12} \Delta q+D_{23} q_{2}, C_{2}=\Delta \omega+$ $D_{23} \Delta q+D_{12} q_{2}, \quad A_{1}=D_{12} \sqrt{4+(\Delta q)^{2}}, \quad B_{1}=D_{23} \sqrt{1+q_{2}^{2}}$, $A_{2}=D_{12} \sqrt{1+q_{2}^{2}}, \quad B_{2}=D_{23} \sqrt{4+(\Delta q)^{2}}, \quad \alpha=\tan ^{-1}\left(\frac{\Delta q}{2}\right)$, $\beta=\tan ^{-1}\left(q_{2}\right)$, and, finally, $\Delta q=q_{2}-q_{1}=q_{2}-q_{3}$ and $\Delta \omega=\omega_{2}-\omega_{1}=\omega_{2}-\omega_{3}$. Note that Eqs. (6) and (7) represent the Adler equation in two variables [46]. Since we are interested in finding the behavior of frequency vs coupling, we first assume that oscillators 2 and 3 are phase entrained, i.e., $\dot{\phi}_{32} \approx 0$; then the beat frequency, $2 \pi\left(\int_{0}^{2 \pi} \frac{d \phi_{12}}{\dot{\phi}_{12}}\right)^{-1}$, between oscillators 1 and 2 is given by

$$
\begin{aligned}
\Omega_{12}= & \left\{\left[\Delta \omega\left(1+\kappa D_{12}\right)-\sin \phi_{32} D_{23}\right]^{2}\right. \\
& \left.-4 D_{12}^{2}\left(1+\kappa^{2} \Delta \omega^{2}\right)\right\}^{1 / 2} .
\end{aligned}
$$

Now, in order to test our hypothesis that a nonmonotonic dependence of the frequency difference on the coupling strength, arising due to a positive covariance of natural frequency and shear, is responsible for the emergence of weak-winner PS, we define $q_{j}=\kappa \omega_{j}, j=1,2,3$, where $\kappa$ is just a scaling constant. This relation ensures that there is a positive covariance between $\omega$ and $q$ when $\kappa>0$, which is needed for APS to manifest. Substituting $q_{j}=\kappa \omega_{j}$ and $D_{23}=0.024$ into Eq. (8), it can be shown that $\Omega_{12}$ is a nonmonotonic function of $D_{12}$ if and only if $\kappa>0$ (see Appendix A), which is further confirmed by a numerical solution [Figs. 4(c) and 4(d)]. Note that the numerically obtained phase diagram of the complex Stuart-Landau system [cf. Figs. 4(a) and 4(b)] represented by Eq. (4) looks very similar to that of the population dynamical system [cf. Fig. 2(a) and 2(c)] represented by Eqs. (1a)-(1c). For a negative covariance, i.e., $\kappa<0$, we find all the regimes [Fig. 4(a)] which are also present in Fig. 2(a) including relay synchronization. However, for positive covariance, i.e., $\kappa>0$, we obtain quite prominent regions of weak-winner PS [Fig. 4(b)]. This demonstrates clearly that the presence of APS leads to weak-winner phase synchronization.

\section{IMPLICATION FOR NETWORKS}

As demonstrated earlier, the weak-winner phenomenon is quite generic with respect to the nature of the dynamics of the individual oscillators. However, one might be tempted to think about another aspect of generality, which is topology. In other words, does this phenomenon hold true for (a) a larger number of oscillators and (b) more complex network topologies?
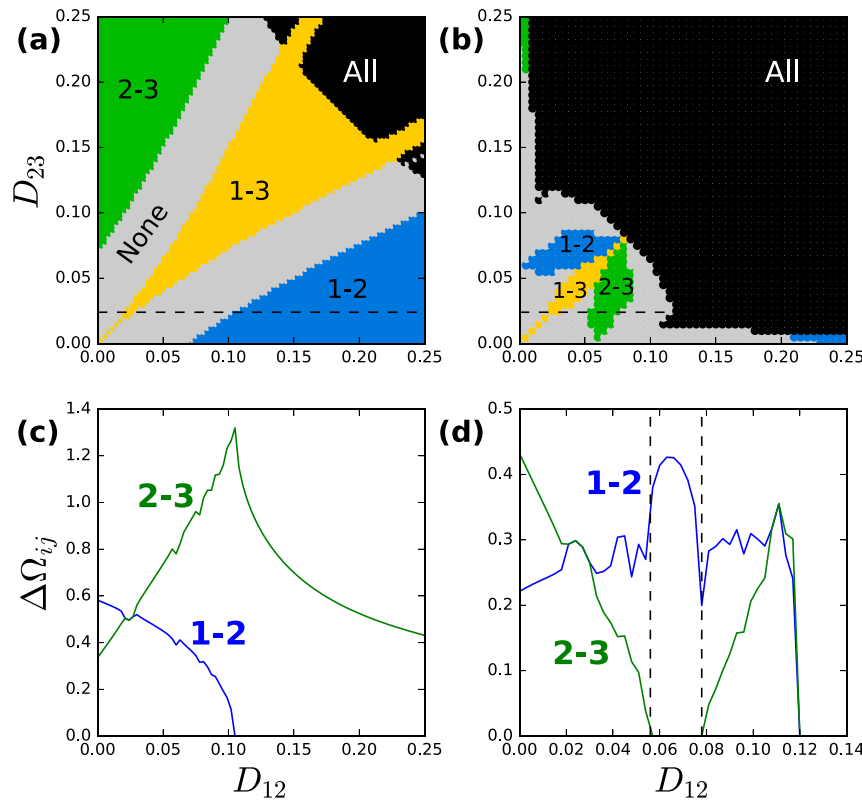

FIG. 4. (a) and (b) Parameter space plots for Stuart-Landau equations showing, as indicated by the labels (and colors), regions of $D_{12}$ and $D_{23}$ values for oscillator pairs in phase synchrony or not. (c) and (d) show the variation of mean relative frequency of pairs 1-2 and 2-3, as labeled (and colored), with $D_{12}$ for a fixed value of $D_{23}=0.024$ [along the horizontal dashed line in Fig. 4(a)]. Here, $\omega_{1,3}=1.2, \omega_{2}=0.949$, and $[(a)$ and (c) $] \kappa=-5$ or [(b) and (d)] $\kappa=5$.

Though the full answer to these questions is beyond the scope of this paper, we present here the first step in this direction by discussing a number of weak-winner patterns that would emerge in larger networks.

Specifically, we construct a setup of four coupled oscillators arranged in such a way that the new setup can be treated as a combination of our old three-oscillator system plus an external fourth oscillator coupled to it, as depicted in the sketch shown in Fig. 5.

In this setup, our three-oscillator system (shaded region in Fig. 5) can serve as a network motif, and the fourth oscillator encapsulates the mean-field contribution of a larger network. To demonstrate the validity of weak-winner phase synchronization in the presence of an external coupling, $D_{24}$ in our case, we simulate the system composed of four Stuart-Landau oscillators arranged in the setup shown in Fig. 5. For this setup the observed distinct weak-winner patterns are sketched in Fig. 6(b), which shows that for larger networks, not only a pair of weakly linked oscillators can synchronize but also a subset of all weakly linked oscillator pairs can synchronize.

For the four-oscillator setup, regions of these distinct weakwinner patterns in the three-dimensional coupling space are presented in Fig. 7. The blue-colored region corresponds to a single pair of oscillators exhibiting weak-winner phase synchronization, while the red-colored region corresponds to a chain (of length 3 ) of oscillators exhibiting weak-winner phase synchronization.

With even larger networks, the number of ways in which weak-winner phase synchronization can manifest would 


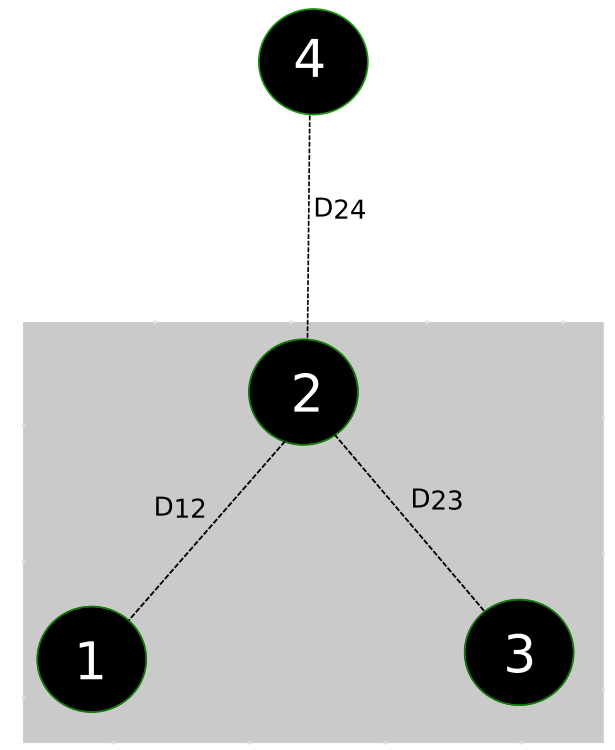

FIG. 5. Sketch for realizing the weak-winner phenomenon in complex networks where our original three-oscillator system (shaded region) is acting as a network motif and the fourth oscillator is acting as a mean-field contribution from the rest of the network.

increase further, giving rise to a wide range of interesting synchronization patterns.

\section{DISCUSSION AND CONCLUSION}

The current approach to the phenomenon of phase synchronization in coupled oscillator systems focuses essentially on the interplay of coupling and frequency detuning between the oscillators. However, this approach often overlooks the crucial role played by nonisochronicity (shear) — an intrinsic property of an individual oscillator-in shaping the emergent collective

(a)
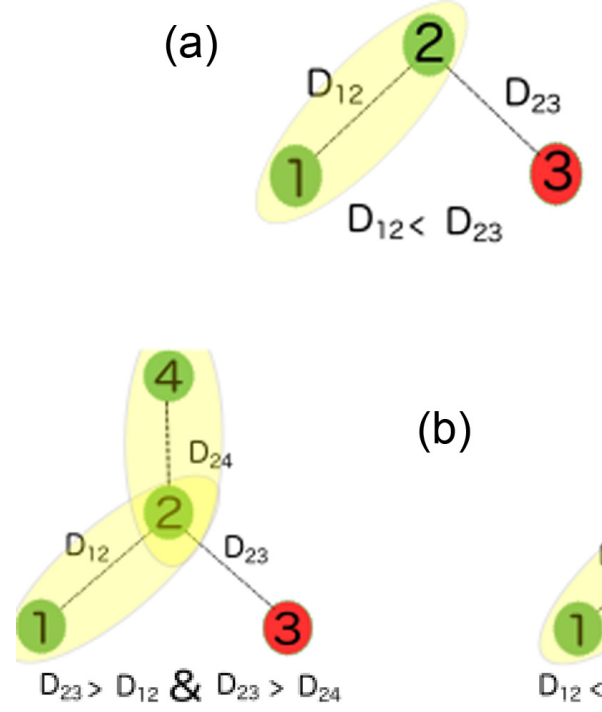

(b)

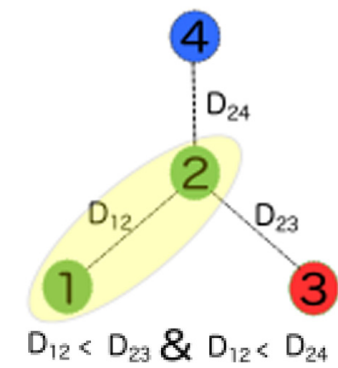

FIG. 6. Distinct number of weak-winner phase synchronization patterns that are observed with a network of (a) three oscillators and (b) four oscillators. Nodes of the same color (shaded in yellow) are phase synchronized, while the other nodes are desynchronized. dynamics of the system. For instance, the mechanism behind the emergence of the counterintuitive weak-winner PS cannot be explained through the current approach. In this context, our study not only offers the underlying mechanism behind weak-winner PS but also sheds some light on the generic question, How does shear influence the phase dynamics? The emergence of weak-winner PS is a phenomenon for which we anticipate potential applications to a large class of problems where phase synchrony is desirable or in some cases undesirable. For example, in the case of the three-patches-of-wildlife equations we used, the coupling strengths correspond to the migration rate of predator and prey species and can be interpreted as movement corridors connecting different patches of wildlife [47]. As a conservation strategy, the design of movement corridors should be such that we are able to control the migration intensity of species so that it does not become too low to risk local extinction or too high to risk global extinction due to synchronization of populations [48-50]. However, the presence of a weak-winner phenomenon could easily make the design of control strategies more difficult as increasing the migration between two patches could induce synchrony among the other patches with weaker migration, which is clearly an undesirable consequence.

Although we have shown in Sec. IV that the emergence of weak-winner PS in larger complex networks could display several interesting patterns of phase synchrony, we have barely scratched the surface of potential applications or problems that might come up. One particularly important problem that we could envisage is related to oscillator networks with shear diversity [51]. The heterogeneity in shear and frequency can induce frustration in the oscillatory system, and this could result in metastable states (weak-winner-like) similar to that of spin-glass systems [52-55].

Furthermore, this study is just an initial step in understanding the role of nonisochronicity (shear) in shaping the synchronization behavior of coupled oscillators. For further analysis, we need a better understanding of the precise functional relationship between system parameters and the resulting nonisochronicity, i.e., How is shear determined by the system parameters of an oscillator? Additionally, although having derived sufficient conditions which help in identifying the regions of the parameter space where one observes weak-winner phase synchronization, it still leaves us with a huge parameter space to explore. To address this in more detail, it would be an interesting and challenging task to derive necessary conditions as well.

In summary, we disclose an intriguing type of phase synchronization in a chain of three coupled oscillators in which the weakly coupled oscillators achieve synchrony while the strongly coupled ones do not. Three key ingredients are needed for weak-winner phase synchronization to manifest itself: (a) a set of coexisting weak links and strong links implying heterogeneity in the coupling, (b) a set of oscillators having different natural frequency implying heterogeneity in the oscillator dynamics, and (c) nonmonotonic behavior of the oscillators' phase difference with coupling implying the presence of anomalous phase synchronization. Furthermore, we have shown how the emergence of this unexpected kind of synchronous behavior can be explained in terms of anomalous phase synchronization, arising from a complex 


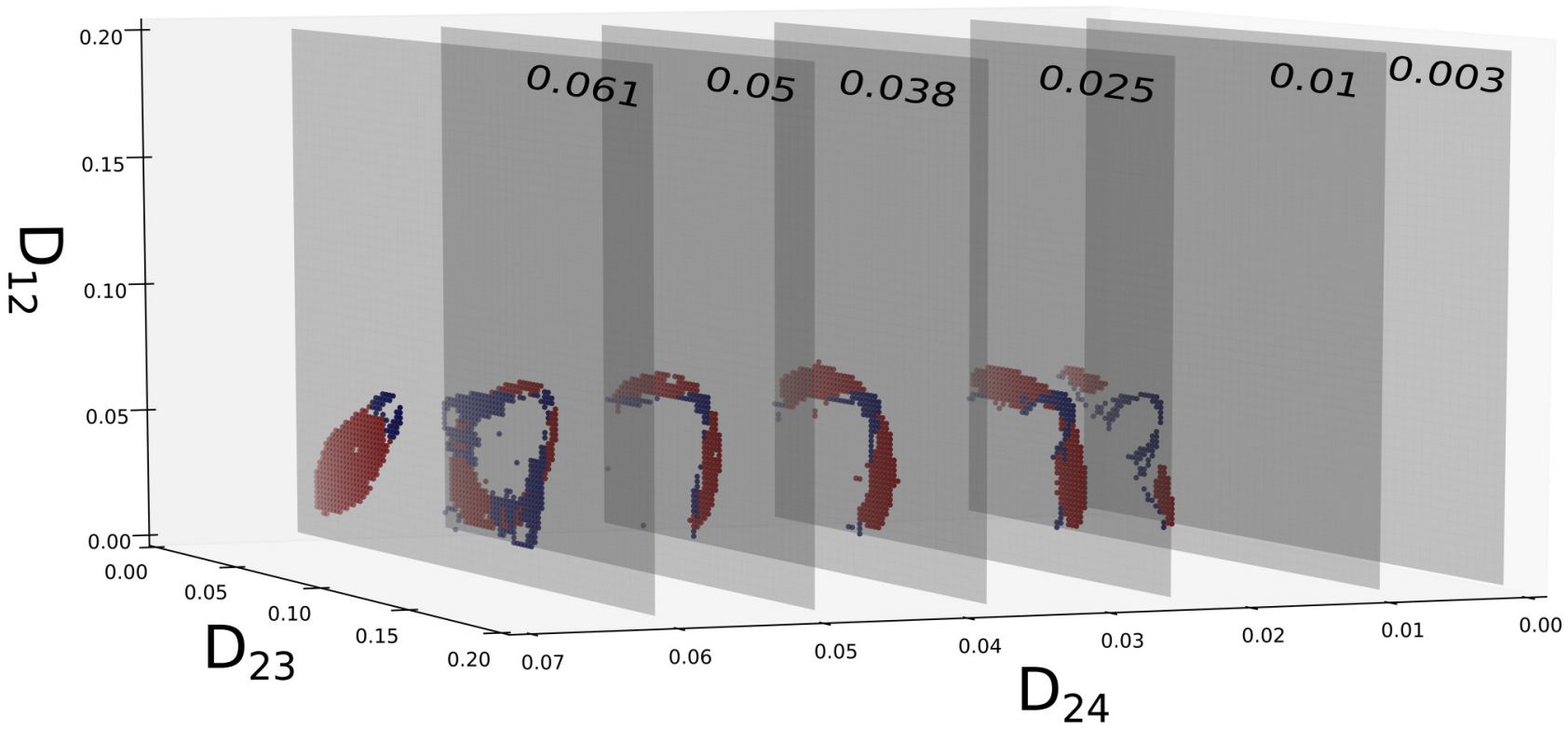

FIG. 7. Regions of weak-winner phase synchronization in a four-oscillator setup (as shown in Fig. 5) with our original three oscillators acting as a network motif. As $D_{24}$ is varied, two distinct regions of weak-winner PS (blue and red colors) are manifested over a significant range of $D_{24}$ values, as shown by parallel planes each corresponding to a fixed value of $D_{24}$.

interplay between shear and natural frequencies of the oscillators. The fact that shear and natural frequency are intrinsic properties of every oscillator makes the manifestation of the weak-winner phenomenon quite generic. We have validated it by considering oscillators exhibiting different dynamical behaviors such as limit cycle and chaotic dynamics. Some potential applications of weak-winner phase synchronization could include, among others, lasers [25,56], communication systems [57], and neuronal systems [58,59]. Lastly, we believe that the mechanism underlying the weak-winner phenomenon would open up a new direction of thinking about the role of nonisochronicity (shear) as a fundamental feature in shaping the emergent dynamics of any coupled oscillator system.

The data used to produce our results is available through GitHub [60].

\section{ACKNOWLEDGMENTS}

The authors would like to thank E. Ott for discussions on a previous version of this manuscript. The simula- tions were performed at the HPC Cluster CARL, located at the University of Oldenburg (Germany) and funded by the Deutsche Forschungsgemeinschaft through its Major Research Instrumentation Programs (Grant No. INST 184/157-1 FUGG) and the Ministry of Science and Culture (MWK) of the Lower Saxony state. A.S. would like to thank the Volkswagen Foundation for financial support (Grant No. 88459). U.F. acknowledges support from the Deutsche Forschungsgemeinschaft (FOR 2716, FE 359-20).

\section{APPENDIX A: BEHAVIOR OF THE FREQUENCY DIFFERENCE $\Omega_{12}$ AS A FUNCTION OF $D_{12}$}

In this Appendix, we demonstrate the existence of anomalous phase synchronization (APS) in our model system [Eq. (4)] by establishing the nonmonotonic behavior of $\Omega_{12}$ as a function of $D_{12}$. We start by expanding Eq. (8) as

$$
\Omega_{12}=\sqrt{\left[2 \kappa \Delta \omega\left(\Delta \omega-D_{23} \sin \phi_{32}\right)\right] D_{12}-4 D_{12}^{2}+\left[\Delta \omega^{2}-2 \Delta \omega \sin \phi_{32} D_{23}+\left(\sin \phi_{32} D_{23}\right)^{2}\right]}
$$

In deriving Eq. (8), we assumed $\dot{\phi}_{32} \approx 0$, which holds true for an interval $\left[D_{12}^{1}, D_{12}^{2}\right]$ [see Fig. 4(d)]. Therefore, for this interval, $\sin \phi_{32}$ becomes a constant. Also, since we are interested in finding the behavior of $\Omega_{12}$ as $D_{12}$ is varied, we keep $D_{23}$ fixed at a value of 0.024 [as shown by the horizontal dashed line in Fig. 4(b)]. Rearranging all the constant terms yields

$$
\Omega_{12}=\sqrt{-4 D_{12}^{2}+\mu D_{12}+v}
$$

where $\mu=2 \kappa\left(0.063-0.006 \sin \phi_{32}\right)$ and $v=(0.063-$ $\left.0.012 \sin \phi_{32}\right)+0.0006 \sin ^{2} \phi_{32}$. Since the function of the relative frequency is quadratic in coupling strength, we can check if an extremum exists in the interval $\left[D_{12}^{1}, D_{12}^{2}\right]$, which would confirm the presence of nonmonotonicity. Therefore the problem now reduces to finding the extremum of $g\left(D_{12}\right)=-4 D_{12}^{2}+\mu D_{12}+v$, which gives us $D_{12}=\frac{\mu}{8}$. Observe that the value of $D_{12}$ is positive (valid solution) if 


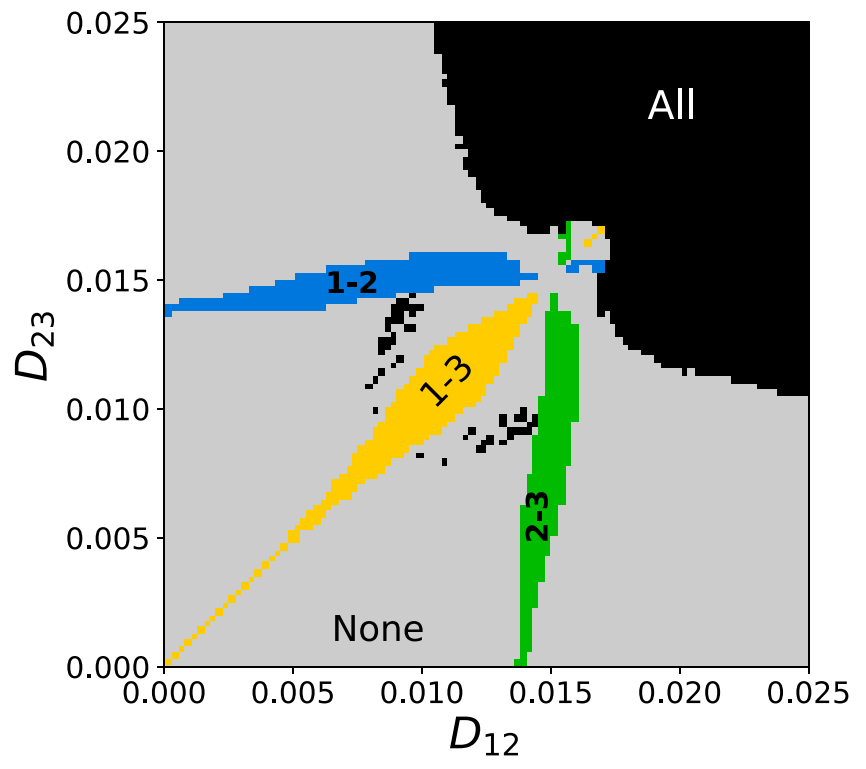

FIG. 8. Parameter space plots showing, as indicated by the labels (and colors), regions of $D_{12}$ and $D_{23}$ values for oscillator pairs in phase synchrony or not. Parameters are $\mathbf{a}=(1,1,1)$ and $\mathbf{b}=$ $(1.0,0.92,1.0)$.

and only if $\mu>0$, which holds true when $\kappa>0$, and this validates our claim that APS exists only for $\kappa>0$.

\section{APPENDIX B: COUPLED VAN DER POL OSCILLATORS}

Here, we present the phase diagram of three coupled van der Pol oscillators exhibiting limit cycle oscillations. The coupling setup used here is the same as in the model of chaotic oscillators given by Eqs. (1a)(1c) in the main text. The governing equations are thus represented by

$$
\begin{aligned}
& \dot{x}_{i}=y_{i}+\sum_{i=1}^{3} D_{i j}\left(x_{j}-x_{i}\right), \\
& \dot{y}_{i}=a_{i}\left(1-x_{i}^{2}\right) y_{i}-b_{i}^{2} x_{i}+\sum_{i=1}^{3} D_{i j}\left(y_{j}-y_{i}\right) .
\end{aligned}
$$

To observe the weak-winner phenomenon, we set $a_{i}=$ $\kappa b_{i}$ with $\kappa=4.0 \forall i=1,2,3$ and $\mathbf{b}=[1,0.92,1]$. Islands of weak-winner solutions (green and blue regions) can be clearly seen in Fig. 8.

\section{APPENDIX C: INTUITION BEHIND ANOMALOUS PHASE SYNCHRONIZATION}

The aim of this Appendix is to promote an intuitive understanding of anomalous phase synchronization (APS). First we introduce the concept of the isochron (shear), which is essentially the dependence of rotation speed on amplitude. Formally, isochrons are defined as a family of curves in phase space where all points on each curve represent a unique phase [61]. To demonstrate this, we take the Stuart-Landau oscillator given by

$$
\dot{z}=z\left[1+i(\omega+q)-(1+i q)|z|^{2}\right],
$$
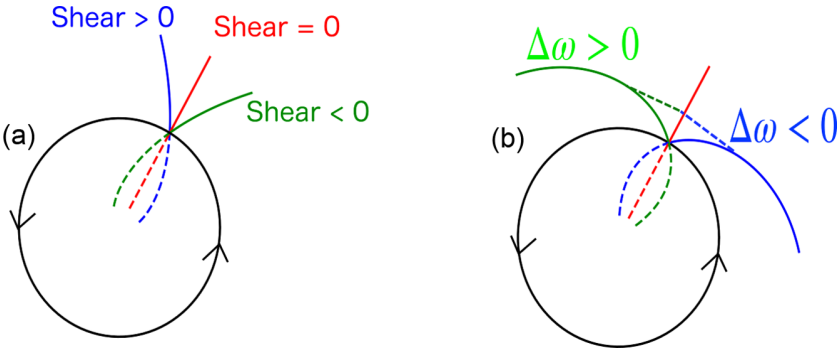

FIG. 9. (a) Isochron and (b) frequency variation with amplitude $\omega(r)$, of a Stuart-Landau oscillator [Eq. (C1)] for positive (blue), negative (green), and zero shear (red) as drawn on polar coordinates $(r, \theta)$. The black curve represents the limit cycle solution of the oscillator with $r=1$.

which in polar coordinates becomes

$$
\begin{gathered}
\dot{r}=r\left(1-r^{2}\right), \\
\dot{\theta}=\omega+q\left(1-r^{2}\right) .
\end{gathered}
$$

This oscillator has a stable limit cycle solution at $r=1$. The phase can be defined in the neighborhood of the limit cycle attractor as [62] $\phi=\theta-q \ln r$, which on the limit cycle becomes just " $\theta$ " as $r=1$. Therefore a typical isochron representing a constant phase $\left(\phi^{*}\right)$ is described as

$$
I_{\phi^{*}}=\phi^{*}=\theta-q \ln r .
$$

When $q=0$, the isochron has no radial component, which means that rotation speed is independent of the position in the neighborhood of the limit cycle. In Fig. 9(a), we show how isochrons change as shear is introduced in the system. For positive shear $(q>0)$, the oscillator's instantaneous frequency increases (decreases) as we move radially inwards (outwards) from the limit cycle. This change in frequency is captured by $\Delta \omega=q\left(1-r^{2}\right)$ as shown in Fig. 9(b). However, for negative shear $(q<0)$, the system gets slower (faster) as it moves inwards (outwards) from the limit cycle. Note that fast and slow are always relative to the case of zero shear $(q=0)$, where the frequency is independent of amplitude.

Now, we extend this picture to two diffusively coupled Stuart-Landau oscillators having frequencies $\omega_{1}$ and $\omega_{2}$, respectively, with $\omega_{1}<\omega_{2}$. Furthermore, we set $q_{i}=\kappa \omega_{i}$ for $i=1,2$, so that $\operatorname{Cov}[q, \omega]$ can be positive, negative, or zero depending on the values of $\kappa$. As before, the relative change in the frequency of the oscillator due to shear is measured by $\Delta \omega_{i}\left(r_{i}\right)$. However, in this setup, shear for an oscillator is not just a constant but depends on its natural frequency $\omega$. To illustrate the effect of shear on the resulting behavior of the coupled system, we consider the three following cases:

(1) $\kappa=O$ (shearless). This is a trivial case where neither oscillator experiences any shear. Here, the diffusive coupling would have a trivial impact on the dynamics; that is, increasing the coupling would slow down the fast oscillator and speed up the slow one until both oscillators lock to a common frequency and start oscillating synchronously.

(2) $\kappa>0$ (positive shear). In this case, both oscillators experience different shear $\left(q_{1,2}=\kappa \omega_{1,2}\right)$. Since $\omega_{1}<\omega_{2}$, we have $q_{1}<q_{2}$. The frequency variation for both oscillators is shown in Fig. 10 marked by their respective colors. For 


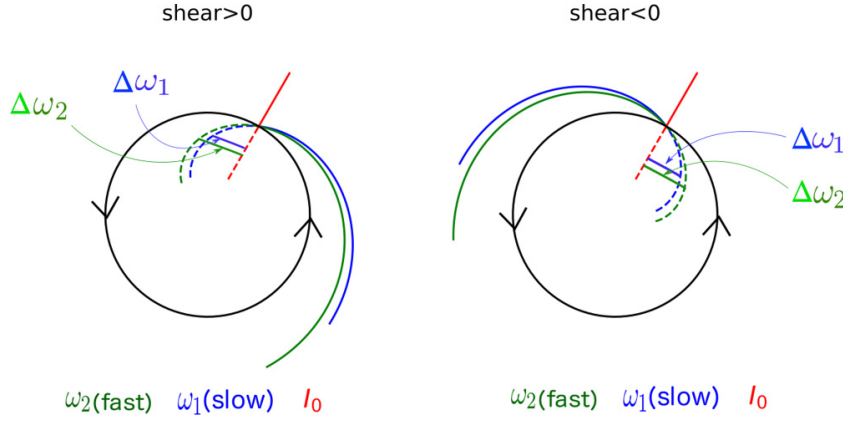

FIG. 10. Frequency variation with amplitude for two diffusively coupled Stuart-Landau oscillators for positive $(\kappa>0)$ and negative $(\kappa<0)$ shear as drawn on polar coordinates $(r, \theta)$. The instantaneous frequency curves corresponding to fast and slow oscillators are represented by green and blue colors, respectively. The red one represents the shearless case. The black curve represents the limit cycle solution with $r=1$ for the uncoupled oscillator.

positive shear, both oscillators speed up but by different amounts $\left(\Delta \omega_{1}<\Delta \omega_{2}\right)$ when they move inwards, away from the limit cycle. It is worth noting here that due to coupling the oscillators are always pushed inwards as shown by the variation of mean amplitude as a function of coupling strength (Fig. 11).

Therefore, for lower coupling strengths, the oscillators are almost always inside the limit cycle, and then the shear comes into play, which in this case widens their initial frequency difference. Eventually, for high enough coupling, the oscillators are pulled back to follow the limit cycle where the effect of shear vanishes and they manage to synchronize. This is essentially the mechanism behind APS.

(3) $\kappa<0$ (negative shear). The explanation for the case of negative shear is quite similar to that of positive shear except here the frequency variation (Fig. 10, right panel) is such that both the oscillators slow down, with the faster one slowing down by a larger amount than the slow one.

Therefore, in contrast to the previous case of positive shear, the weak coupling would shrink their initial frequency difference. This means that negative shear synchronizes the system at a coupling strength even lower than that of zero shear.

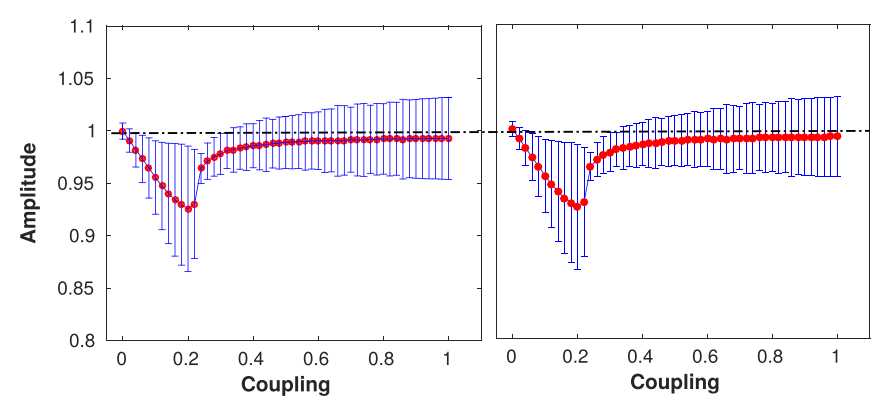

FIG. 11. Variation of amplitudes (left, $r_{1}$; right, $r_{2}$ ) with coupling strength for two diffusively coupled Stuart-Landau oscillators. The natural frequencies of the oscillators are $[0.95,1.2]$ and $\kappa=5$. Red points indicate mean values, and error bars (blue) represent the standard deviation.
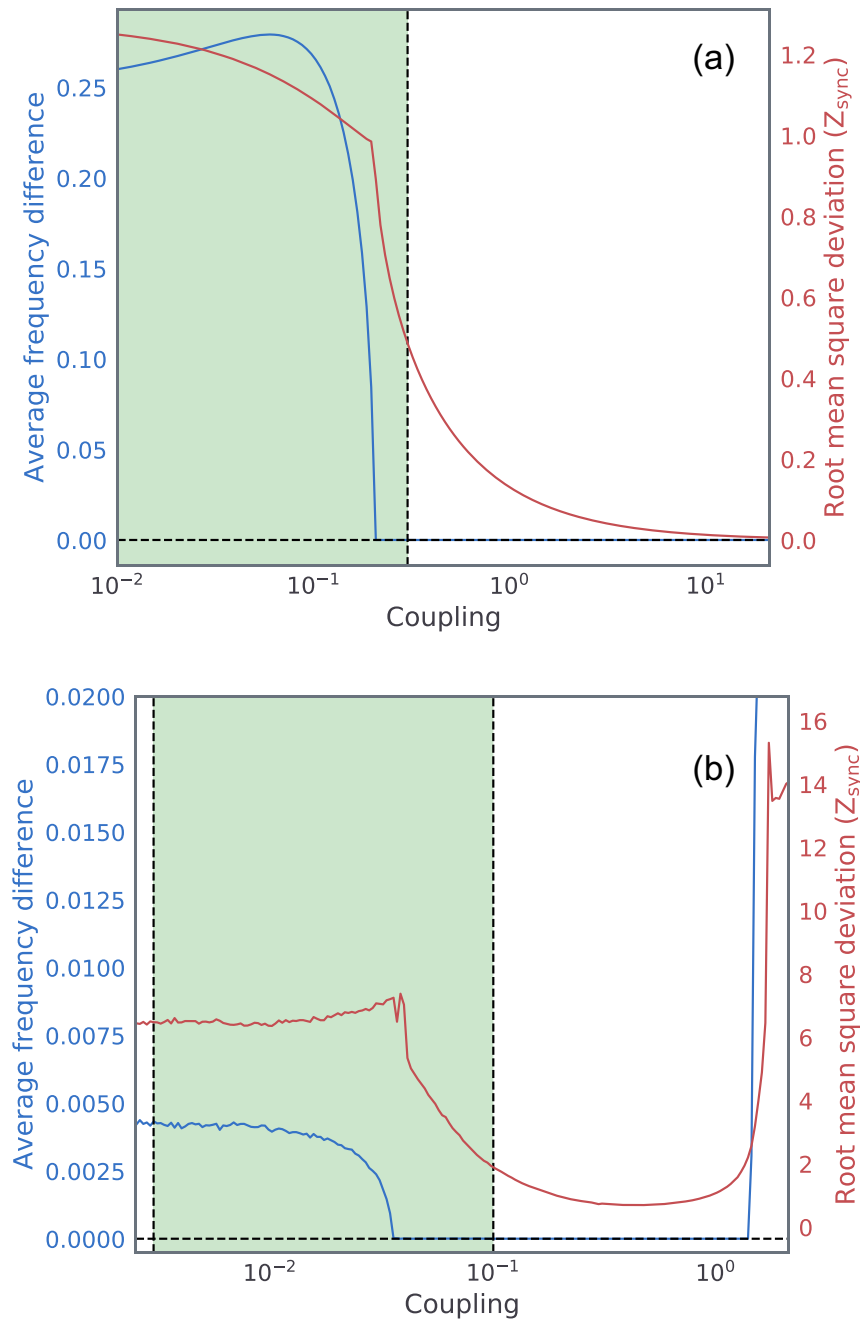

FIG. 12. Variation of the average frequency difference (blue curve) and the root-mean-square deviation $\left(Z_{\text {sync }}\right.$, red curve) with the coupling strength for two diffusively coupled oscillators. (a) Coupled limit cycle oscillators [Eq. (D1)]. (b) Coupled chaotic food web model [Eqs. (D2a)-(D2c)]. The green-shaded areas depict the range of coupling strengths considered in this paper.

\section{APPENDIX D: CONNECTION TO SHORT-WAVELENGTH BIFURCATION}

One might suspect that weak-winner phase synchronization arises as a consequence of a short wavelength bifurcation (SWB) of the type discovered for diffusively coupled oscillators [36]. Briefly, SWB is a bifurcation of the synchronized dynamics residing on the invariant synchronization manifold in a system of coupled identical oscillators, where upon increasing coupling strength, the system loses synchrony. It occurs when the eigenvalue corresponding to the smallest spatial Fourier mode becomes positive. As a consequence of this bifurcation, the system can only be synchronized for a bounded range of coupling strengths.

At first, this could suggest an explanation of weak-winner PS due to the fact that in a system with mixed coupling strengths, the weaker coupling is still in the intermediate range (where synchrony is possible) while the stronger coupling is already beyond that range. However, via numerical 
simulations we demonstrate below that the pairs of coupled limit cycle and chaotic oscillators never lose synchronization after its onset, within the investigated range of coupling strengths.

(1) Limit cycle case.

$$
\dot{z}_{j}=z_{j}\left[1+i\left(\omega_{j}+q_{j}\right)-\left(1+i q_{j}\right)\left|z_{j}\right|^{2}\right]+D\left(z_{k}-z_{j}\right) .
$$

(2) Chaotic food web case.

$$
\begin{aligned}
& \dot{x}_{j}=a_{j} x_{j}-\frac{\epsilon_{1} x_{j} y_{j}}{\left(1+k_{1} x_{j}\right)}, \\
& \dot{y}_{j}=-b_{j} y_{j}+\frac{\epsilon_{1} x_{j} y_{j}}{\left(1+k_{1} x_{j}\right)}-\epsilon_{2} y_{j} z_{j}+D\left(y_{k}-y_{j}\right), \\
& \dot{z}_{j}=-c_{j}\left(z_{j}-\zeta_{j}\right)+\epsilon_{2} y_{j} z_{j}+D\left(z_{k}-z_{j}\right),
\end{aligned}
$$

where $j, k=1,2(j \neq k)$ and $D$ is the coupling strength. The system parameters for the limit cycle case are $\omega_{1}=0.949$, $\omega_{2}=1.2$, and $q_{1,2}=\kappa \omega_{1,2}$ with $\kappa=4.0$; for the chaotic oscillator case, they are $a_{1}=a_{2}=1.0, b_{1}=0.9, b_{2}=1.3$, $c_{1}=c_{2}=10.0, \epsilon_{1}=0.25, \epsilon_{2}=1.0$, and $\zeta_{1}=\zeta_{2}=0.006$. We would like to emphasize here that the system parameters of both oscillators are such that there is a slight detuning in the natural frequencies of the oscillators, which essentially means that they are nonidentical in contrast to the case considered by Pecora and Carroll in their master stability approach [36].

For both the systems [Eqs. (D1) and (D2a)-(D2c)], we observe the following quantities as the coupling between the oscillators is varied:

(1) Average frequency difference. This is $\left\langle f_{1}-f_{2}\right\rangle_{t}$, where $f_{1}$ and $f_{2}$ represent the instantaneous frequencies of oscillators 1 and 2, respectively. The average of the frequency difference is taken over time " $t$ steps" after transients have settled. When this quantity approaches zero, we have phase synchronization between oscillators.
(2) Root-mean-square deviation. This quantity measures the extent of complete synchronization in the system. Mathematically, it is given by

$$
Z_{\text {sync }}=\sqrt{\frac{1}{N T} \sum_{t=1}^{T} \sum_{i=1}^{N}\left\|\left(\mathbf{X}_{\mathbf{i}}(\mathbf{t})-\langle\mathbf{X}(\mathbf{t})\rangle_{i}\right)\right\|^{2}},
$$

where $\langle\cdot\rangle_{i}$ is the average over the number of oscillators $N,\|\cdot\|$ is the Euclidean norm, and $1<t<T$ is the time interval after transients have settled. In the case of complete synchronization, i.e., $\mathbf{X}_{\mathbf{1}}=\mathbf{X}_{\mathbf{2}}=\cdots=\mathbf{X}_{\mathbf{N}} \forall t, Z_{\text {sync }} \rightarrow 0$ asymptotically.

The simulation results are presented in Fig. 12, and there are several things to be noted here:

(i) For the complete range of coupling strengths used in this paper (green-shaded regions in Fig. 12), the system composed of two oscillators never loses phase synchrony (indicated by the blue curve) once established, as the coupling is increased. This clearly rules out the involvement of SWB for both limit cycle and chaotic oscillators.

(ii) For coupling strengths beyond our studied window, we anticipate that as coupling is increased, the oscillator's amplitude will also tend to synchronize, which is reflected by a decrease in $Z_{\text {sync }}$. In the case of the limit cycle system [Fig. 12(a)], upon increasing coupling further, the system does not lose its synchrony. However, for the chaotic coupled oscillator case [Fig. 12(b)], the system undergoes a loss of synchrony upon increasing coupling strength, which might be a signature of SWB. Please note that this happens at coupling values much higher than the ones used in this paper.

(iii) Moreover, while the SWB reported by Pecora and Carroll [36] was found for identical oscillators and identical coupling strengths, the weak-winner PS that we present here emerges only when oscillators are detuned sufficiently and the coupling strengths are not identical.
[1] C. Huygens, Horologium Oscillatorium (Muguet, Paris, 1986).

[2] A. F. Taylor, M. R. Tinsley, F. Wang, Z. Huang, and K. Showalter, Dynamical quorum sensing and synchronization in large populations of chemical oscillators, Science 323, 614 (2009).

[3] B. Ermentrout, M. Pascal, and B. Gutkin, The effects of spike frequency adaptation and negative feedback on the synchronization of neural oscillators, Neural Comput. 13, 1285 (2001).

[4] R. E. Mirollo and S. H. Strogatz, Synchronization of pulsecoupled biological oscillators, SIAM J. Appl. Math. 50, 1645 (1990).

[5] K. Wiesenfeld, P. Colet, and S. H. Strogatz, Synchronization Transitions in a Disordered Josephson Series Array, Phys. Rev. Lett. 76, 404 (1996).

[6] W. Singer, Synchronization of cortical activity and its putative role in information processing and learning, Annu. Rev. Physiol. 55, 349 (1993).

[7] S. H. Strogatz, Sync: How Order Emerges from Chaos in the Universe, Nature, and Daily Life (Hachette, London, 2012).
[8] L. M. Pecora and T. L. Carroll, Synchronization in Chaotic Systems, Phys. Rev. Lett. 64, 821 (1990).

[9] L. Kocarev and U. Parlitz, Generalized Synchronization, Predictability, and Equivalence of Unidirectionally Coupled Dynamical Systems, Phys. Rev. Lett. 76, 1816 (1996).

[10] M. G. Rosenblum, A. S. Pikovsky, and J. Kurths, Phase Synchronization of Chaotic Oscillators, Phys. Rev. Lett. 76, 1804 (1996).

[11] A. Pikovsky, M. Rosenblum, and J. Kurths, Synchronization: A Universal Concept in Nonlinear Sciences, Cambridge Nonlinear Science Series Vol. 12 (Cambridge University Press, Cambridge, 2003).

[12] X. F. Wang, Complex networks: Topology, dynamics and synchronization, Int. J. Bifurcation Chaos Appl. Sci. Eng. 12, 885 (2002).

[13] J. A. Acebrón, L. L. Bonilla, C. J. Pérez Vicente, F. Ritort, and R. Spigler, The Kuramoto model: A simple paradigm for synchronization phenomena, Rev. Mod. Phys. 77, 137 (2005). 
[14] J. Thévenin, M. Romanelli, M. Vallet, M. Brunel, and T. Erneux, Resonance Assisted Synchronization of Coupled Oscillators: Frequency Locking without Phase Locking, Phys. Rev. Lett. 107, 104101 (2011).

[15] Z. Zheng, G. Hu, and B. Hu, Phase Slips and Phase Synchronization of Coupled Oscillators, Phys. Rev. Lett. 81, 5318 (1998).

[16] B. Blasius, E. Montbrió, and J. Kurths, Anomalous phase synchronization in populations of nonidentical oscillators, Phys. Rev. E 67, 035204(R) (2003).

[17] E. Montbrió and B. Blasius, Using nonisochronicity to control synchronization in ensembles of nonidentical oscillators, Chaos 13, 291 (2003).

[18] S. K. Dana, B. Blasius, and J. Kurths, Experimental evidence of anomalous phase synchronization in two diffusively coupled Chua oscillators, Chaos 16, 023111 (2006).

[19] M. Wickramasinghe and I. Z. Kiss, Synchronization of electrochemical oscillators with differential coupling, Phys. Rev. E 88, 062911 (2013).

[20] B. Blasius, Anomalous phase synchronization in two asymmetrically coupled oscillators in the presence of noise, Phys. Rev. E 72, 066216 (2005).

[21] I. T. Tokuda, S. K. Dana, and J. Kurths, Detecting anomalous phase synchronization from time series, Chaos 18, 023134 (2008).

[22] H. Nakao, T. Yanagita, and Y. Kawamura, Phase-Reduction Approach to Synchronization of Spatiotemporal Rhythms in Reaction-Diffusion Systems, Phys. Rev. X 4, 021032 (2014).

[23] E. Rosa, E. Ott, and M. H. Hess, Transition to Phase Synchronization of Chaos, Phys. Rev. Lett. 80, 1642 (1998).

[24] R. Breban and E. Ott, Phase synchronization of chaotic attractors in the presence of two competing periodic signals, Phys. Rev. E 65, 056219 (2002).

[25] R. McAllister, R. Meucci, D. DeShazer, and R. Roy, Competition between two frequencies for phase synchronization of a chaotic laser, Phys. Rev. E 67, 015202(R) (2003).

[26] B. Schelter, M. Winterhalder, R. Dahlhaus, J. Kurths, and J. Timmer, Partial Phase Synchronization for Multivariate Synchronizing Systems, Phys. Rev. Lett. 96, 208103 (2006).

[27] I. Nishikawa, N. Tsukamoto, and K. Aihara, Switching phenomenon induced by breakdown of chaotic phase synchronization, Phys. D (Amsterdam) 238, 1197 (2009).

[28] I. Fischer, R. Vicente, J. M. Buldú, M. Peil, C. R. Mirasso, M. C. Torrent, and J. García-Ojalvo, Zero-Lag Long-Range Synchronization Via Dynamical Relaying, Phys. Rev. Lett. 97, 123902 (2006).

[29] L. V. Gambuzza, M. Frasca, L. Fortuna, and S. Boccaletti, Inhomogeneity induces relay synchronization in complex networks, Phys. Rev. E 93, 042203 (2016).

[30] R. Gutiérrez, R. Sevilla-Escoboza, P. Piedrahita, C. Finke, U. Feudel, J. M. Buldú, G. Huerta-Cuellar, R. Jaimes-Reátegui, Y. Moreno, and S. Boccaletti, Generalized synchronization in relay systems with instantaneous coupling, Phys. Rev. E 88, 052908 (2013).

[31] B. Blasius, A. Huppert, and L. Stone, Complex dynamics and phase synchronization in spatially extended ecological systems, Nature (London) 399, 354 (1999).
[32] N. J. Gotelli, A Primer of Ecology (Sinauer, Sunderland, MA, 1995).

[33] O. Rössler, An equation for continuous chaos, Phys. Lett. A 57, 397 (1976).

[34] For the range of parameter values used in this paper, the individual oscillators of our system remain phase coherent. However, the methods we employ for detecting and analyzing phase synchronization are applicable to phase noncoherent oscillators as well, as long as adequate techniques are used for obtaining their phases. See, for example, R. Follmann, E. Rosa, Jr., and E. E. N. Macau, Phys. Rev. E 83, 016209 (2011).

[35] J. Dormand and P. Prince, A family of embedded Runge-Kutta formulae, J. Comput. Appl. Math. 6, 19 (1980).

[36] L. M. Pecora and T. L. Carroll, Master Stability Functions for Synchronized Coupled Systems, Phys. Rev. Lett. 80, 2109 (1998).

[37] M. Rosenblum and A. Pikovsky, Two types of quasiperiodic partial synchrony in oscillator ensembles, Phys. Rev. E 92 012919 (2015).

[38] A. A. Temirbayev, Y. D. Nalibayev, Z. Z. Zhanabaev, V. I. Ponomarenko, and M. Rosenblum, Autonomous and forced dynamics of oscillator ensembles with global nonlinear coupling: An experimental study, Phys. Rev. E 87, 062917 (2013).

[39] J. Norris, The closing of Arnol'd tongues for a periodically forced limit cycle, Nonlinearity 6, 1093 (1993).

[40] S. Ehrich, A. Pikovsky, and M. Rosenblum, From complete to modulated synchrony in networks of identical Hindmarsh-Rose neurons, Eur. Phys. J.: Spec. Top. 222, 2407 (2013).

[41] C. van Vreeswijk, Partial synchronization in populations of pulse-coupled oscillators, Phys. Rev. E 545522 (1996).

[42] P. Mohanty and A. Politi, A new approach to partial synchronization in globally coupled rotators, J. Phys. A: Math. Gen. 39, L415 (2006).

[43] L. P. Gor'kov, Microscopic derivation of the Ginzburg-Landau equations in the theory of superconductivity, Sov. Phys. JETP 36, 1364 (1959).

[44] I. S. Aranson and L. Kramer, The world of the complex Ginzburg-Landau equation, Rev. Mod. Phys. 74, 99 (2002).

[45] Y. Kuramoto, Chemical Oscillations, Waves, and Turbulence, Springer Series in Synergetics Vol. 19 (Springer, New York, 2012).

[46] R. Adler, A study of locking phenomena in oscillators, Proc. IRE 34, 351 (1946).

[47] S. A. Cushman, K. S. McKelvey, and M. K. Schwartz, Use of empirically derived source-destination models to map regional conservation corridors, Conserv. Biol. 23, 368 (2009).

[48] A. Liebhold, W. D. Koenig, and O. N. Bjørnstad, Spatial synchrony in population dynamics, Annu. Rev. Ecol. Evol. Syst. 35, 467 (2004).

[49] M. D. Holland and A. Hastings, Strong effect of dispersal network structure on ecological dynamics, Nature (London) $\mathbf{4 5 6}$ 792 (2008).

[50] D. J. D. Earn, S. A. Levin, and P. Rohani, Coherence and conservation, Science 290, 1360 (2000).

[51] E. Montbrió and D. Pazó, Shear Diversity Prevents Collective Synchronization, Phys. Rev. Lett. 106, 254101 (2011). 
[52] M. Mézard, G. Parisi, N. Sourlas, G. Toulouse, and M. Virasoro, Nature of the Spin-Glass Phase, Phys. Rev. Lett. 52, 1156 (1984).

[53] M. Nixon, E. Ronen, A. A. Friesem, and N. Davidson, Observing Geometric Frustration with Thousands of Coupled Lasers, Phys. Rev. Lett. 110, 184102 (2013).

[54] D. Iatsenko, P. V. McClintock, and A. Stefanovska, Glassy states and super-relaxation in populations of coupled phase oscillators, Nat. Commun. 5, 4118 (2014).

[55] C.-U. Choe, J.-S. Ri, and R.-S. Kim, Incoherent chimera and glassy states in coupled oscillators with frustrated interactions, Phys. Rev. E 94, 032205 (2016).

[56] D. J. DeShazer, R. Breban, E. Ott, and R. Roy, Detecting Phase Synchronization in a Chaotic Laser Array, Phys. Rev. Lett. 87, 044101 (2001).
[57] B. Jovic, Synchronization Techniques for Chaotic Communication Systems (Springer, Berlin, 2011).

[58] J. Fell and N. Axmacher, The role of phase synchronization in memory processes, Nat. Rev. Neurosci. 12, 105 (2011).

[59] U. Feudel, A. Neiman, X. Pei, W. Wojtenek, H. Braun, M. Huber, and F. Moss, Homoclinic bifurcation in a HodgkinHuxley model of thermally sensitive neurons, Chaos 10, 231 (2000).

[60] A. Choudhary, Weak-winner phase synchronization, https:// github.com/anshu957/WeakWinner (2021).

[61] A. T. Winfree, The Geometry of Biological Time, Interdisciplinary Applied Mathematics Vol. 12 (Springer, New York, 2001).

[62] E. Montbrió i Fairen, Synchronization in ensembles of nonisochronous oscillators, Ph.D. thesis, Universität Potsdam, 2004. 